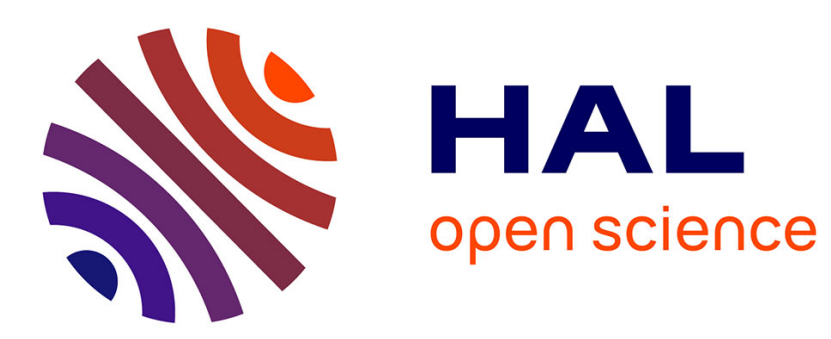

\title{
Choisir des cas exemplaires: la strategic litigation face aux discriminations
}

Aude Lejeune, Jean-François Orianne

\section{To cite this version:}

Aude Lejeune, Jean-François Orianne. Choisir des cas exemplaires: la strategic litigation face aux discriminations. Déviance et Société, 2014, 10.3917/ds.381.0055 . halshs-02177587

\section{HAL Id: halshs-02177587 https://shs.hal.science/halshs-02177587}

Submitted on 12 Jul 2019

HAL is a multi-disciplinary open access archive for the deposit and dissemination of scientific research documents, whether they are published or not. The documents may come from teaching and research institutions in France or abroad, or from public or private research centers.
L'archive ouverte pluridisciplinaire HAL, est destinée au dépôt et à la diffusion de documents scientifiques de niveau recherche, publiés ou non, émanant des établissements d'enseignement et de recherche français ou étrangers, des laboratoires publics ou privés. 


\section{CHOISIR DES CAS EXEMPLAIRES : LA STRATEGIC LITIGATION FACE AUX DISCRIMINATIONS}

Aude Lejeune et Jean-François Orianne

Médecine \& Hygiène | «Déviance et Société »

2014/1 Vol. 38 | pages 55 à 76

ISSN 0378-7931

Article disponible en ligne à l'adresse :

https://www.cairn.info/revue-deviance-et-societe-2014-1-page-55.htm

Distribution électronique Cairn.info pour Médecine \& Hygiène.

(C) Médecine \& Hygiène. Tous droits réservés pour tous pays.

La reproduction ou représentation de cet article, notamment par photocopie, n'est autorisée que dans les limites des conditions générales d'utilisation du site ou, le cas échéant, des conditions générales de la licence souscrite par votre établissement. Toute autre reproduction ou représentation, en tout ou partie, sous quelque forme et de quelque manière que ce soit, est interdite sauf accord préalable et écrit de l'éditeur, en dehors des cas prévus par la législation en vigueur en France. Il est précisé que son stockage dans une base de données est également interdit. 


\section{Choisir des cas exemplaires: la Strategic litigation face aux discriminations*}

\author{
Aude Lejeune \\ CNRS/CERAPS \\ Jean-François Orianne \\ Université de Liège
}

Le recours stratégique aux cours et tribunaux, dit strategic litigation, fait partie des modes d'action des organismes de promotion de l'égalité des chances en Europe. Centré sur le niveau organisationnel, le présent article se propose d'examiner les modalités et conditions de recours à cette pratique dans le cadre de la lutte contre les discriminations en Belgique, les critères et arguments qui permettent de choisir des cas considérés comme exemplaires, ainsi que les transformations potentielles des usages des cours et tribunaux et de l'arène judiciaire qui en découleraient.

La législation européenne en matière de discrimination invite les pays membres à créer des organismes publics nationaux de promotion de l'égalité des chances afin d'assurer l'application des lois relatives à la lutte contre les discriminations. Leur mission est double: accompagner les plaignants dans le processus de dépôt de plaintes et promouvoir le recours au corpus législatif pertinent par les citoyens, fonctionnaires, professionnels et entreprises confrontés à ou impliqués dans des situations de discriminations. Dans le cadre de leurs missions, les organismes ainsi créés sont susceptibles de recourir à une pratique qu'ils qualifient de strategic litigation ${ }^{1}$, que l'on pourrait traduire par «usage stratégique des cours et tribunaux». À travers la défense d'un cas individuel et particulier, ces organismes cherchent à utiliser les juridictions dans le but de transformer des politiques ou législations existantes. À partir de l'analyse de cette pratique en Belgique, nous montrerons que toutes les affaires ne sont pas nécessairement portées devant les cours et tribunaux. En effet, en

* Cette recherche a été réalisée dans le cadre d'un financement du Fonds spécial de la recherche de la Communauté française de Belgique. Nous remercions Rachel Brahy, Vincent-Arnaud Chappe, Patrick Charlier, Cécile Guillaume, Virginie Guiraudon, Olgierd Kety, Andrée Lemaître, Michel Pasteel, Sophie Pochic et les participants du RT13 du Congrès de l'AFS à Grenoble pour leurs commentaires et remarques sur une version antérieure de ce texte. Les propos tenus ici n'engagent cependant que leurs auteurs. Nous remercions également Pierre-Yves Jortay pour sa participation à l'enquête de terrain.

1 Notons que la notion strategic litigation est devenue depuis peu une expression indigène: elle est couramment utilisée par les acteurs, aussi bien au sein des organismes de promotion de l'égalité que par les avocats ou les associations de défense des droits. 
plus de son coût, le procès n'est pas le seul moyen de défendre les droits des citoyens. Les organismes de promotion de l'égalité belges privilégient d'ailleurs les modes extra-judiciaires de résolution des conflits mais, dans certains cas, ils décident d'entreprendre une action en justice. L'orientation vers la strategic litigation implique donc de choisir, parmi les cas individuels de discrimination que traitent ces organismes, les dossiers qui sont «exemplaires» et qui pourront permettre aux magistrats de créer une jurisprudence nouvelle dans certains domaines particuliers du droit ${ }^{2}$. La strategic litigation repose sur le postulat d'une influence du droit sur la réalité sociale: une transformation législative devrait permettre, à plus long terme, de faire évoluer les pratiques des différents acteurs du monde du travail, tant parmi les employeurs que les travailleurs ou les syndicats.

À ce jour, peu de travaux se sont attachés à examiner les différents facteurs qui influencent le recours à la strategic litigation par des organisations de défense des droits (Vanhala, 2009). Le rôle des agences gouvernementales dans la promotion des droits des citoyens fait partie des zones d'ombre de cette littérature. Centré sur le niveau organisationnel, cet article se propose de combler en partie cette lacune en se penchant sur les modalités et conditions de recours à cette pratique comme mode d'action de lutte contre les discriminations en Belgique, sur les critères et arguments qui permettent de choisir des cas considérés comme exemplaires, ainsi que sur les transformations potentielles des usages des cours et tribunaux et de l'arène judiciaire qui en découleraient ${ }^{3}$.

Si les États-Unis et, plus généralement, les pays de droit anglo-saxon, ont depuis longtemps recours aux cours et tribunaux en vue de produire des transformations de l'ordre politique et social (1), cette pratique a été introduite récemment en Belgique, notamment dans le cadre de la lutte contre les discriminations et constitue une rupture forte avec la tradition légale belge (2). Partant d'une enquête de terrain auprès de juristes qui travaillent dans les organismes de promotion de l'égalité, nous mettrons en lumière les tensions qui entourent le processus de sélection des dossiers ainsi que les arguments et registres de justification auxquels ils se réfèrent (3). Au travers de ce processus, les juristes développent une stratégie de promotion des droits qui passe par le recours aux cours et tribunaux belges, ce qui invite à réfléchir à la potentielle émergence de nouveaux usages de l'arène judiciaire en Belgique.

Cet article s'appuie sur une recherche empirique qui traite des discriminations dans l'emploi en Belgique. Le travail de terrain s'est principalement déroulé entre octobre 2010 et janvier 2011. Le matériau empirique se compose essentiellement d'entretiens et d'observations dans les deux organismes chargés de la lutte contre les discriminations en Belgique: le Centre pour l'égalité des chances et la lutte contre le racisme (CECLR) et l'Institut pour l'égalité des femmes et des hommes (IEFH), tous deux implantés à Bruxelles. Il s'agit précisément d'entretiens auprès de juristes et collaborateurs de ces organismes en charge des «dossiers emploi» $(\mathrm{N}=10)$, d'entretiens avec les plaignants $(\mathrm{N}=8)$, d'observations d'interactions entre les juristes et les plaignants $(\mathrm{N}=6)$ et entre les juristes eux-mêmes, lors de réunions d'équipe $(\mathrm{N}=4)$. En outre, nous avons réalisé une analyse documentaire: consultation de dossiers de-

2 La sélection de dossiers exemplaires ne constitue donc qu'une part de leur activité, à côté du traitement individuel des signalements ou plaintes.

3 Le présent article place la focale sur les pratiques des collaborateurs des organismes de promotion de l'égalité, ainsi que sur leurs interactions avec d'autres intervenants de professions judiciaires, de syndicats et d'organisations non gouvernementales, ainsi qu'avec les requérants. 
puis le dépôt de la plainte jusqu'au jugement $(\mathrm{N}=26)$, examen de rapports d'activité, de notes de travail internes aux services, etc. Enfin, nous avons procédé à des entretiens complémentaires d'avocats et de magistrats en charge de dossiers de cette nature $(\mathrm{N}=7)$. Lors de ces entretiens et du dépouillement des dossiers, nous avons cherché à comprendre les critères que les conseillers juridiques mettent en évidence pour sélectionner les cas qui bénéficieront d'un soutien juridique et financier de la part du CECLR ou de l'IEFH.

\section{Les juridictions comme arène politique: une spécificité des pays de common law?}

Traditionnellement, les pays de common law, tels que le Royaume-Uni ou les États-Unis, sont plus enclins au développement de la strategic litigation que les pays d'Europe continentale. En effet, leur système de droit est davantage ouvert aux changements établis par la jurisprudence que dans les pays de droit civil, où les sources formelles du droit, telles que les codes, prédominent. La littérature de sciences sociales sur l'usage stratégique de l'arène judiciaire s'est donc d'abord développée aux États-Unis. Selon ces travaux, les cours et tribunaux joueraient un rôle capital dans l'évolution des normes et seraient plus enclins que les pouvoirs législatifs et exécutifs à prendre des décisions qui ont une influence sociale et politique dans le domaine de la promotion des droits (Handler et al., 1978; McCann, 1994; García Villegas, 2009). Bien que ce constat soit nuancé par de nombreux sociologues et juristes qui manifestent un scepticisme grandissant à l'égard du potentiel du droit à changer la société (Galanter, 1974; Scheingold, 1974; Rosenberg, 1993), les professionnels du droit ont souvent été étudiés comme des acteurs politiques aux États-Unis, ce qui n'est pas le cas en Europe où le droit et le politique ont été considérés comme deux champs séparés de recherche (Commaille, 2000; Lejeune, 2011). Le courant du Judicial politics a mis en évidence le fait que les magistrats sont amenés à interpréter les sources du droit, y compris la constitution, ce qui leur confère un rôle politique de première importance (Commaille, Dumoulin, 2009; Miller, 2009). Le courant du Cause lawyering a quant à lui étudié les avocats qui utilisent l'arène judiciaire pour défendre des causes politiques (Sarat, Scheingold, 1998; Israël, 2001).

Ultérieurement, les travaux sur les usages stratégiques des cours et tribunaux ont été importés sur le vieux continent dans le cadre d'études des modalités d'action politique des groupes d'intérêt au sein de l'Union européenne (Conant, 2006; Case, Givens, 2010). Certains se sont intéressés aux ressources dont disposent les groupes pour recourir stratégiquement aux cours et tribunaux (Alter, Vargas, 2000), d'autres ont mis en avant les processus de cadrage des problèmes publics (Vanhala, 2009). Récemment, ces réflexions, auxquelles cette communication contribue, ont été introduites dans le paysage de la sociologie francophone dans le cadre d'analyses des usages sociaux et politiques du droit et de la justice (Vauchez, 2001; Agrikoliansky, 2003; Pelisse, 2005; Revillard, 2007; Israël, 2009; Guillaume, Pochic, 2012). Malgré ce foisonnement récent de travaux sur le niveau européen, peu d'attention a été portée à cette stratégie de recours aux cours et tribunaux au sein d'agences gouvernementales. Notons que la notion de strategic litigation, qui englobe toutes les pratiques de recours au litige à des fins stratégiques, est plus large que celle de 
cause lawyering, focalisée exclusivement sur la défense de causes politiques. Elle permet de prendre en compte les contraintes spécifiques rencontrées par les conseillers juridiques qui travaillent au sein des agences de promotion de l'égalité, notamment parce qu'ils sont contraints de combiner, dans leur activité quotidienne, la promotion de l'égalité en tant que principe universel, d'une part et la défense des minorités ou des catégories socialement dominées ciblées par les législations en matière de non-discrimination, d'autre part (Lejeune, 2014).

Une question traverse l'ensemble de ces travaux, francophones comme anglophones. Les pays européens, en l'occurrence la Belgique, connaissent-ils aussi ce recours aux cours et tribunaux à des fins stratégiques? Assistons-nous à un mouvement de transnationalisation des pratiques du droit qui tendrait à diversifier les modalités de recours aux tribunaux? Au contraire, ce mouvement contribuerait-il, notamment, à la suite de la création d'un espace de justice européen, à une uniformisation? Telle est l'hypothèse défendue par certains auteurs qui parlent de la montée en puissance d'une régulation à travers le litige dans les pays de l'Union européenne (Kelemen, 2011). Dans le domaine de la lutte contre les discriminations, plusieurs exemples récents vont dans le sens d'un recours stratégique aux juridictions: l'affaire Feryn, explicitée infra, portée devant le tribunal du travail d'Anvers puis devant la Cour européenne de Justice a contribué à faire évoluer la définition d'une situation de discrimination en Belgique puis en Europe, tandis que l'affaire Francovich en Italie a contribué à renforcer la protection des travailleurs discriminés lors de la faillite de leur entreprise. Il faut cependant souligner ici que ces usages militants des cours et tribunaux ne sont pas neufs, en Belgique pas plus que dans les autres pays européens. Bien avant l'apparition de la thématique de la discrimination, certains avocats et magistrats s'efforçaient déjà d'utiliser et de transformer le droit en vue de défendre certains idéaux politiques, notamment en droit du travail (Lochak, 1989; Willemez, 2003). Cependant, la nouveauté réside aujourd'hui dans la généralisation de cette stratégie au sein des agences de promotion de l'égalité et l'élaboration d'une réflexion, au niveau national et européen, sur les enjeux de cette stratégie de recours à la justice.

\section{La strategic litigation dans la lutte contre les discriminations en Belgique}

L'analyse de la lutte contre les discriminations en Belgique présente un intérêt majeur lorsque l'on souhaite mettre en évidence les usages potentiellement politiques des cours et tribunaux. Les législations dans cette matière sont relativement récentes; elles sont le fruit d'une réflexion élaborée à un niveau supranational - et, dans une moindre mesure, au niveau des États-nations - et leur mise en œuvre repose, entre autres, sur de nouvelles modalités de sollicitation des juridictions en vue de transformer le droit. Revenir brièvement sur l'histoire de ces politiques de non-discrimination en Belgique permet de mettre en évidence les spécificités de ce champ d'intervention publique.

La non-discrimination est une catégorie juridique qui se développe à partir des années 1970 en Belgique et qui s'impose progressivement dans le paysage juridique. D'abord appliquée aux inégalités entre hommes et femmes sur le marché du travail, cette notion va être 
utilisée également à partir des années 1980 pour désigner toute atteinte à l'égalité en raison de l'origine ethnique. Ce n'est cependant que dans les années 2000 que le droit antidiscriminatoire va véritablement prendre son ampleur, sous linjonction directe du droit communautaire, avec la promulgation en 2003 d'une première loi orientée explicitement vers la lutte contre les discriminations (Bribosia, Rorive, 2003) et, le 10 mai 2007, de trois législations, la loi tendant à lutter contre certaines formes de discriminations, la loi tendant à lutter contre la discrimination entre les femmes et les hommes et la loi modifiant la loi du 30 juillet 1981 tendant à réprimer certains actes inspirés par le racisme et la xénophobie.

Ces législations nationales résultent à la fois d'une impulsion externe et d'une impulsion interne (Guiraudon, 2009). D'une part, elles sont le produit d'une incitation communautaire qui invite les États membres à légiférer en la matière ${ }^{4}$. De l'autre, les législations résultent également de l'action d'organismes qui, aux niveaux fédéral et régional, ont contribué à porter et à défendre la construction de la discrimination en tant que problème public en Belgique. Certains professionnels du droit, principalement des avocats du travail, organisés autour d'associations de défense de certaines minorités, telles que les homosexuels ou les travailleurs immigrés, ont créé depuis de nombreuses années des groupes de professionnels du droit avec lesquels les praticiens interagissent, se comparent et définissent des attentes communes et des standards (Mather, 2009, 58).

Plusieurs évolutions importantes résultent des législations belges de 2003 et de 2007 en matière de lutte contre les discriminations (Ringelheim, 2010) ${ }^{5}$. Tout d'abord, les critères de discrimination ont été élargis afin d'anticiper certaines directives européennes ou de s'aligner sur les exigences définies par le droit communautaire. Par la suite, la définition de la discrimination a fait l'objet d'un nouvel élargissement en intégrant les notions telles que la discrimination indirecte $e^{6}$ ou inconsciente (Sturm, 2001) ou l'annonce publique de discrimination, sans pour autant qu'il y ait de victime individuelle identifiable. Enfin, les difficultés d'accès au droit et à la justice des victimes de discriminations ont été prises en considération. Concrètement, le dépôt de plainte et la mobilisation du droit par les victimes ont été simplifiés par le biais du renversement de la charge de la preuve ${ }^{7}$ et par la création ou la désignation d'organismes spécifiques de traitement de ces questions, le Centre pour l'égalité des chances et la lutte contre le racisme (CECLR) et l'Institut pour l'égalité des femmes et des hommes (IEHF) ${ }^{8}$.

4 Directives européennes 2000/43/CE du 29 juin 2000 relatives à la mise en œuvre du principe de l'égalité de traitement entre les personnes sans distinction de race ou d'origine ethnique et 2000/78/CE du 27 novembre 2000 portant création d'un cadre légal en faveur de l'égalité de traitement en matière d'emploi et de travail.

5 Pour le cas français, voir Fassin, 2002; Bereni, Chappe, 2011.

6 Il faut noter que la loi du 7 mai 1999 (sur l'égalité de traitement entre hommes et femmes en ce qui concerne les conditions de travail, l'accès à l'emploi et aux possibilités de promotion, l'accès à une profession indépendante et les régimes complémentaires de sécurité sociale) distinguait déjà la discrimination directe et la discrimination indirecte en les définissant. En outre, l'ancienne version de la loi du 30 juillet 1981 visait également les discriminations indirectes.

7 Désormais, les trois législations précisent: «Au plaignant, il appartiendra de produire des faits ou tout autre élément de preuve susceptible de faire naître une présomption de discrimination; au défendeur, il appartiendra alors de renverser cette présomption ».

8 La directive 2000/43/CE impose la création d'un organisme indépendant chargé d'assurer l'accompagnement et la défense des victimes de discrimination. 
Ces deux organismes ont pour objectif de promouvoir la lutte contre les discriminations par le biais d'interventions auprès de victimes individuelles et d'actions à un niveau structurel et collectif. Tous deux ont pour mission d'agir en justice dans les litiges auxquels pourrait donner lieu l'application des lois pénales et des autres lois qui ont spécifiquement pour objet la garantie de l'égalité (voir encadré précisant leurs missions et composition en annexe). Les conseillers juridiques qui y travaillent ont pour la plupart une formation en droit ou en sciences sociales. Ceux qui ont une expérience professionnelle antérieure ont travaillé en tant qu'avocats dans des cabinets spécialisés en droit des étrangers ou dans des associations militantes spécialisées dans la défense des droits. Un petit nombre est issu de l'administration. Ils ont comme point commun de revendiquer un engagement dans la lutte contre les discriminations et la promotion des droits.

\section{Choisir des «cas exemplaires": sélectionner et solliciter}

Tous les dossiers signalés ${ }^{9}$ auprès des organismes de promotion de l'égalité ne conduisent pas à la formulation d'une revendication à l'encontre de la personne ou de l'organisme à l'origine du comportement vécu comme une injustice. De même, toutes les revendications ne se transforment pas en débat contradictoire entre la partie requérante et la partie défenderesse. Tous les recours ne se transforment pas non plus en procès devant les juridictions civiles ou pénales (Miller, Sarat, 1981). À chacune des étapes de la construction des plaintes, les juristes procèdent à une sélection des dossiers. Ce processus ne les conduit pas pour autant à «abandonner» les dossiers qui ne vont pas en justice. Souvent, les conseillers cherchent des solutions alternatives de résolution du conflit, les victimes de discrimination ne souhaitant d'ailleurs pas à tout prix ester en justice. La judiciarisation de la plainte, c'est-à-dire sa constitution en litige et le recours aux tribunaux pour régler ce litige, ne doit pas être confondue avec sa juridicisation, c'est-à-dire sa qualification en termes juridiques (Pelisse, 2009). Dans certains cas néanmoins, le manque d'éléments probants et de preuves matérielles peut conduire à la fermeture d'un dossier.

On privilégie d'abord les modes de résolution des conflits extra-judiciaires. L'action en justice est longue et n'est pas toujours adaptée aux attentes des personnes. Même si, au début, les personnes pensent que c'est l'action en justice qui va résoudre tout, quand on écoute leurs attentes, on se rend compte que ce n'est pas l'action en justice, c'est que les faits cessent, qu'ils obtiennent des excuses ou ce à quoi ils croient avoir droit. Et malheureusement l'action en justice n'a pas toujours cet objectif-là (Conseillère juridique, CECLR).

9 Peut être défini comme signalement ou notification toute sollicitation du CECLR ou de l'IEFH par un individu, qu'il s'estime lui-même victime de discrimination ou qu'il estime être ou avoir été témoin d'un comportement discriminatoire à l'encontre d'une tierce personne. Lorsqu'un signalement donne lieu à un suivi, un dossier est créé. Seuls les signalements qui entrent dans les compétences du CECLR ou de l'IEFH donnent lieu à la création d'un dossier (voir annexe). 
Une procédure en justice, ça a un coût pour la victime, sur le plan psychologique. C'est très long et ce n'est pas toujours ce que la victime veut, parfois elle attend une réponse rapide, pour pouvoir passer à autre chose, rechercher un autre emploi. Du coup, il faut peser le pour et le contre d'une action en justice (Conseiller juridique, CECLR).

Les dossiers qui ont été choisis pour être défendus en justice nous intéressent plus particulièrement ici parce qu'ils sont considérés, à un moment donné, comme des « cas exemplaires », révélateurs de problèmes récurrents rencontrés par les travailleurs qui s'estiment victimes de discrimination.

On n'a pas vraiment une liste de critères, c'est plutôt au cas par cas qu'on décide d'ester en justice ou pas. En gros, on rencontre la personne en première ligne, en deuxième ligne, puis si on pense qu'il faut aller en justice, on soumet cette idée en réunion déquipe. Et là, on débat. Et ça peut durer très longtemps! (Conseillère juridique, CECLR).

Lorsqu'on est confronté à un dossier qui est susceptible de faire jurisprudence, alors on réfléchit pour voir si on a des éléments suffisants (Conseiller juridique, CECLR).

Dans leur pratique quotidienne, les juristes ne disposent pas d'une liste arrêtée de critères objectifs, qui s'appliqueraient de façon mécanique, pour déterminer les dossiers qui doivent faire l'objet d'un suivi approfondi par le CECLR ou l'IEFH. Ils sont amenés, lors de réunions d'équipe, à présenter leurs dossiers et à suggérer, pour certains d'entre eux, une intervention en justice. Ce processus de sélection des dossiers place les juristes face à l'obligation de justifier et d'argumenter leurs choix, aux yeux de leurs collègues, de la direction de leur organisation, mais aussi des plaignants et des autres intervenants potentiels, l'avocat ou le juriste du syndicat. Au sein même des équipes, il n'existe pas a priori de consensus lors de la prise de décision d'entamer une procédure en justice. La grille d'évaluation qu'ils mobilisent est construite au cours de leurs interactions, dans l'action de sélection des dossiers. Deux tensions principales apparaissent régulièrement: 1) la volonté de soutenir la victime et de réparer l'injustice subie à un niveau individuel par une indemnisation rapide se heurte au souhait de défendre un intérêt collectif - celui de l'organisation ou de la société civile - via le recours aux cours et tribunaux; 2) la promotion de l'égalité des personnes entre en tension avec l'inégalité de traitement des dossiers que le processus de sélection génère. Quels sont, dès lors, les critères de recherche et de sélection de cas exemplaires pour lesquels leur organisation entreprend une action en justice? Quelles sont les implications de cette décision? Comment justifient-ils leurs choix?

Les stratégies qu'ils mettent en place et les décisions qu'ils prennent sont construites dans l'interaction avec leurs pairs, avec les avocats ou avec les syndicats. À partir des multiples critères auxquels ils se réfèrent pour évaluer la pertinence d'une action en justice, nous proposons de mettre en évidence différents types d'arguments invoqués pour choisir des cas jugés exemplaires. Ces différents arguments peuvent être éclairés à partir d'une grammaire de la justification (Boltanski, Thévenot, 1991) ${ }^{10}$ afin de rendre compte,

10 En s'appuyant sur les travaux de Garfinkel, en particulier le concept d'accountability, Luc Boltanski et Laurent Thévenot ont formulé une étiologie des justifications mobilisées par les acteurs, en situation d'épreuves ou de 
d'une part, des enjeux en tension qui traversent ces organisations et, d'autre part, du travail quotidien de mise en cohérence, d'articulation, autour d'une stratégie collective - la strategic litigation - susceptible de faire consensus autour de certains dossiers. L'usage que nous faisons ici des travaux de Boltanski et Thévenot doit être précisé: notre étude de cas ne vise pas à analyser la manière dont un compromis se forme en situation de conflit ou de lutte autour de la définition d'un bien commun; elle cherche à rendre compte de l'élaboration d'une stratégie collective de sélection et de recherche de «cas exemplaires ». C'est moins la dispute au sein des équipes que la dissonance cognitive et normative induite par l'incertitude des situations de travail et la multiplicité d'enjeux organisationnels difficilement conciliables qui alimente cette activité de délibération et de justification à laquelle se livrent les conseillers juridiques. Ce cadrage théorique permettra d'interroger les conditions pragmatiques d'un jugement équilibré et d'un arbitrage sur les cas, au sein des équipes de conseillers, entre diverses contraintes normatives ou appuis conventionnels, tant dans le processus de sélection que de recherche de cas exemplaires.

\section{Le processus de sélection}

Les appuis conventionnels sur lesquels ils s'appuient peuvent être répartis en quatre groupes: la légalité (a), la visibilité (b), le professionnalisme (c) et le connexionnisme (d).

\section{Une intervention juste au regard du droit}

Lorsqu'ils sont confrontés à un cas nouveau, les conseillers juridiques se réfèrent tout d'abord aux textes légaux. Une distinction de traitement n'est pas considérée en soi comme une discrimination. Pour être qualifiée de discriminatoire, la distinction doit porter sur l'un des critères protégés par la loi. Aujourd'hui, la législation belge a déterminé dix-neuf critères, sur la base desquels la discrimination est interdite, tels que la nationalité, la couleur de peau, le genre, l'âge ou l'orientation sexuelle ${ }^{11}$. La qualification par le juriste d'une injustice en tant que discrimination n'est pas mécanique mais dépend des faits et de l'appréciation qu'il porte sur la situation.

Quand une personne vous raconte qu'elle a été discriminée, il faut voir si le comportement qu'elle considère comme discriminatoire se rapporte à un trait défini par la loi. Certaines personnes estiment qu'elles n'ont pas été traitées de manière égale en raison de leur appartenance à un cercle de collègues qui sont moins bien traités par exemple. Ça ne peut pas être considéré comme une discrimination, sauf si la personne apporte d'autres éléments pour montrer que les membres de ce groupe sont discriminés en raison de leur couleur de peau, de leur sexe ou de tout autre critère défini par la loi (Conseiller juridique, CECLR).

disputes, en fonction de leurs capacités critiques. Six ordres de grandeur ont ainsi été identifiés: la cité inspirée, domestique, de l'opinion, civique, marchande et industrielle. Plus récemment, L. Boltanski et È. Chiapello (1999) ont argumenté l'existence d'une septième cité, dite des réseaux ou par projets.

11 Les dix-neuf critères sont: la nationalité, la prétendue race, la couleur de peau, l'ascendance ou l'origine nationale ou ethnique, lâge, l'orientation sexuelle, les convictions syndicales et religieuses, philosophiques ou politiques, le handicap, le genre, l'état civil, la naissance, l'état de santé, une caractéristique physique ou génétique ou encore l'origine sociale. 
Les organismes de promotion de l'égalité ne sont d'ailleurs compétents que dans le cas de distinctions fondées sur l'un des critères protégés par la loi. Or, toutes les demandes adressées à ces organismes ne s'inscrivent pas dans leur domaine d'action. Les conseillers juridiques sont donc amenés à expliquer aux plaignants les domaines d'action pour lesquels ils sont compétents et, lorsque les requêtes s'inscrivent hors de leur champ d'activité, à renvoyer les personnes vers d'autres organismes.

Lydie est conseillère juridique. Cette après-midi, elle s'occupe des permanences téléphoniques. Elle reçoit les appels de requérants qui lui expliquent leur propre situation d'injustice ou celle qu'endure l'un de leurs proches. Dans de nombreux cas, Lydie est amenée à expliquer la législation et les limites de leur domaine de compétence aux plaignants. Elle explique: "Ce n'est pas évident de faire comprendre aux gens qui vivent mal une situation qu'on ne peut rien faire. Avant ce coup de fil, il y a peutêtre eu des jours et des jours de réflexion avant d'oser prendre son téléphone. Alors on sait que, même si on réoriente, on perd pas mal de monde en chemin, des gens qui ne feront pas d'autres démarches. Souvent, ils ne comprennent pas qu'on ne puisse pas les aider».

Ces organismes de promotion de l'égalité ont conclu des accords de collaboration avec les syndicats, dans un pays où le taux de syndicalisation est relativement élevé (52\% en $2010)^{12}$. Ces accords précisent que, dans les cas où une discrimination paraît avérée et qu'elle touche au domaine de l'emploi et que le travailleur est syndiqué, ce dernier doit d'abord être défendu par son syndicat. Cependant:

Dans certains cas, le syndicat refuse de prendre en charge les frais de justice parce qu'ils disent qu'il n'y a pas assez d'éléments par exemple, alors si c'est vraiment une question importante de discrimination, on réfléchit au rôle que l'on peut jouer (Conseillère juridique, CECLR).

Si ce premier critère de qualification juridique peut paraître objectif, il laisse en réalité une grande marge d'appréciation aux conseillers juridiques. En effet, la qualification d'une injustice en tant que discrimination n'est pas automatique. Dans de nombreux cas, l'expérience vécue par le travailleur pourrait, au regard des législations sur la non-discrimination, être considérée comme relevant de la discrimination et, au regard des législations de protection des travailleurs, être envisagée comme une injustice à l'égard d'un travailleur vulnérable.

Au Centre [CECLR] et à l'Institut [IEFH], ils ont des «lunettes discrimination» comme ils disent. Ils voient tout à travers cette législation, alors que moi, comme d'autres avocats d'ailleurs, je me pose vraiment la question de la plus-value des législations contre les discriminations, par rapport aux législations sociales classiques. Si ça ne permet pas de demander une indemnité plus importante, à quoi bon? C'est une législation à la mode, c'est tout (Avocat spécialisé en droit du travail).

12 La moyenne européenne de syndicalisation est de 34,6\% en 2010 (Terraz, Jaoul-Grammare, 2012). 
Si le fait peut être qualifié de discriminatoire, les juristes se réfèrent à l'état de la jurisprudence dans le domaine, ce qui constitue un deuxième critère de sélection, toujours en référence aux textes légaux. Ils seront plus enclins à défendre un dossier si la jurisprudence en la matière est faible ou inexistante. Les organismes belges ont pour mission de porter en justice des affaires qui permettront de créer une jurisprudence nouvelle qui touche à des questions jusqu'alors ignorées par le droit. Leur action consiste dans ce cas à définir la situation comme problématique et à faire reconnaître l'existence d'un «vide juridique » autour de ces questions (Memmi, 1989). Si les juristes pensent que la législation n'est pas claire sur certains points ou qu'elle ne couvre pas toutes les formes possibles de discrimination, ils auront tendance à privilégier ce dossier-là plutôt qu'un autre. La question de la maternité a, par exemple, fait l'objet de nombreuses clarifications : qu'entend-on notamment par maternité (une fausse-couche peut-elle être associée à une maternité du point de vue de la discrimination?) ou qu'en est-il des conditions de protection de la femme enceinte (une femme enceinte dont le contrat à durée déterminée n'est pas renouvelé entret-elle dans les conditions de protection de la loi contre les discriminations?).

D'abord, on regarde si c'est un point de droit qui doit être tranché, ou en tout cas où il faut qu'on tape sur le clou au niveau belge. [...] Par exemple, on constate qu'il y a un grand sentiment de discrimination au sein du groupe des femmes enceintes, ou des travailleuses qui ont accouché et que, à côté de ça, on n'avait pas beaucoup de dossiers, on n'a pas tellement de jurisprudence vraiment sur la discrimination. Il y a de la jurisprudence sur la protection de la maternité mais pas sur la discrimination (Conseillère juridique, IEFH).

Si le droit communautaire ou la jurisprudence européenne se sont prononcés sur une question mais que le jugement ou l'arrêt n'a pas été traduit en droit belge, les organismes de promotion de l'égalité peuvent inciter à porter l'affaire devant les juridictions, afin de traduire le droit communautaire en droit belge.

Dans certains cas, la loi existe mais elle n'est pas bien comprise ou pas bien interprétée par les magistrats. Donc il faut qu'on arrive à les faire réfléchir sur l'application du droit communautaire. Parce que, dans certains domaines, on a le droit communautaire qui est plus poussé que le droit belge en matière de discrimination. Donc il faut essayer de faire en sorte que les magistrats aillent plus loin, qu'ils aillent voir dans le droit communautaire. Dans le cas de la femme enceinte dont le contrat de travail n'a pas été renouvelé après sa maternité, on a des éléments dans la jurisprudence communautaire. La Cour de Justice avait déclaré que, effectivement, il y avait discrimination dans ce cas-là (Conseillère juridique, IEFH).

Ces deux premiers critères de sélection de cas exemplaires s'inscrivent dans un registre de justification civique (Boltanski, Thévenot, 1991, 231-241). Le caractère officiel des textes de loi et le respect des formes légales constituent les principaux appuis conventionnels de l'action. Le premier critère permet de classer les signalements en trois catégories principales: 1) ceux qui font l'objet d'un traitement en interne (constitution d'un dossier); 2) ceux qui sont transférés à un partenaire (syndicats, associations, avocats spécialisés, etc.); 3) ceux qui sont classés «sans suite». Le second critère permet d'opérer un premier tri, parmi les dos- 
siers constitués en interne, en sélectionnant quelques dossiers «stratégiques » qui doivent être portés à la connaissance de l'équipe. Il permet en outre au conseiller juridique de s'extraire de la particularité du cas, d'une situation d'injustice, pour envisager la défense plus générale du principe de non-discrimination. Précisons que ces critères servent d'arguments lorsque les conseillers sont amenés à justifier leurs choix auprès du public, de la hiérarchie ou de leurs collègues. Ce registre de justification civique apparait comme «allant de soi » compte tenu de la mission officielle de ces organismes: promouvoir l'égalité. Cependant, l'analyse approfondie de dossiers, l'observation de réunions d'équipe ainsi que les entretiens individuels réalisés auprès de ces juristes font apparaître d'autres critères (que ceux définis par la loi) et registres de justification.

\section{Une intervention reconnue}

Un troisième critère de sélection, lié aux deux premiers, a trait à la définition de domaines prioritaires; ces priorités varient d'année en année pour chaque organisme. Si le dossier s'inscrit dans les enjeux stratégiques et les priorités politiques et stratégiques du CECLR ou de l'IEFH, ils sont d'autant plus attentifs à suivre ce dossier-là plutôt qu'un autre. Ainsi, récemment, la lutte contre les comportements homophobes sur le lieu de travail faisait partie, entre autres, des priorités du CECLR, tandis que l'IEFH privilégiait les actions visant à lutter contre les discriminations au travail liées à la maternité.

Parfois, on essaie de faire des focus, par exemple cette année ça va être l'emploi, ou cette année ça va être les homosexuels. Donc forcément quand il y a des dossiers qui portent sur l'une de ces matières-là, on essaie de les porter en justice (Conseillère juridique, CECLR).

Ces domaines d'intervention ont été définis comme prioritaires soit parce que les membres de ces organisations pensent qu'il est pertinent de modifier une loi ou une politique qui viole les droits fondamentaux, soit parce qu'ils estiment qu'il faut s'assurer qu'une loi est appliquée correctement. Plusieurs travaux ont montré que la probabilité de produire un changement significatif dans les législations et politiques publiques existantes dépend de plusieurs critères: l'existence d'un groupe d'intérêt organisé, ayant un mandat spécifique et défini, doté de ressources suffisantes pour pouvoir défendre plusieurs affaires liées entre elles (Bouwen, McCown, 2007, 429). Ici, la sélection de domaines prioritaires permet donc, à un moment donné, de créer un effet de nombre qui renforce les capacités d'action et limpact potentiel des actions entreprises. Plus généralement, le ciblage de domaines d'action prioritaire permet aux organismes de défense des droits des travailleurs d'augmenter leur visibilité au sein de l'espace public, notamment à travers les médias. Cette dimension représente un enjeu essentiel pour l'IEFH et le CECLR car il est susceptible de permettre une augmentation des demandes adressées par les travailleurs qui s'estiment victimes d'une injustice. La visibilité de leurs actions permet de véhiculer des normes et des valeurs de non-discrimination et, par là-même, favorise la création de nouvelles demandes. Ils participent ainsi à la socialisation juridique de groupes sociaux plus larges et c'est par ce biais qu'ils sont susceptibles de légitimer leur propre existence. 
La focalisation sur certains domaines prioritaires, qui évoluent au fil des années, témoigne d'un autre ordre de grandeur: la réputation ou le renom de l'organisme. Le monde de l'opinion (Boltanski, Thévenot, 1991, 222-230) est ici convoqué par les juristes du CECLR et de l'IEFH pour sélectionner des cas susceptibles d'accroître la visibilité de leur travail au sein de l'espace public, notamment via les médias, de convaincre le grand public, les décideurs politiques, le monde associatif et le monde du travail, de l'intérêt et de la pertinence de leur intervention - quitte à renoncer au caractère privé d'un cas singulier, de l'expérience subjective d'une situation d'injustice. Cette hiérarchie des priorités, largement informelle, entre en tension avec la mission officielle de ces organismes par l'inégalité de traitement des cas qu'elle induit. Par ailleurs, ce focus permet de créer une masse critique de dossiers qui renforce l'efficacité de chacune des actions entreprises, ce qui nous conduit à un autre ordre de valeur, le professionnalisme des conseillers.

\section{Une intervention performante}

Il y a certaines contraintes à prendre en compte pour que la mobilisation des cours et tribunaux porte ses fruits. Ainsi, un quatrième critère de sélection des dossiers est la solidité et la robustesse des éléments qui les constituent. Il faut que le dossier contienne suffisamment de preuves et d'éléments tangibles: il s'agit d'un principe fondamental de toute action en justice (Perrouty, Rorive, 2005). Ensuite, il faut que le dossier ne soit pas ambigu et qu'il permette de créer une jurisprudence positive. Un dossier qui manque d'éléments de preuves pourrait conduire à créer une jurisprudence négative qui aurait des répercussions néfastes sur les actions entreprises ultérieurement par les conseillers et sur les recours des travailleurs. La sélection des dossiers fait naître, comme l'a par ailleurs montré Vincent-Arnaud Chappe (2010) dans son analyse de la permanence juridique d'une association antiraciste en France, une tension entre la volonté de soutenir la victime et le réalisme de la procédure à entreprendre. Les conseillers juridiques sont souvent confrontés à des récits de situations qu'ils perçoivent comme injustes mais face auxquelles ils se sentent impuissants pour venir en aide aux personnes car celles-ci n'apportent pas suffisamment de preuves tangibles.

On ne veut surtout pas créer de jurisprudence négative, donc si le dossier est beaucoup trop faible sur le plan de la preuve, on ne prend pas le risque. Parfois, on sent que le dossier ne tient pas et donc on préfère attendre de tomber sur un "bon dossier», bien constitué, plutôt que de risquer de créer une jurisprudence négative (Conseillère juridique, IEFH).

Ces critères relatifs à la solidité ou à la robustesse des éléments qui constituent un dossier s'inscrivent, comme celui de l'effet de nombre, dans un registre industriel de justification (Boltanski, Thévenot, 1991, 252-262): l'évaluation ex ante de la performance de l'intervention est ici au cœur du processus de sélection de cas. Un professionnel du droit «compétent» se doit de tester, de mesurer, grâce à un raisonnement juridique rigoureux, les moyens et ressources dont il dispose, l'effectivité d'une preuve, l'exactitude d'informations produites, en clair de maîtriser le processus de constitution d'un dossier. Le monde industriel est ici convoqué pour se protéger d'un risque professionnel majeur: l'inefficacité d'une action en justice et le caractère contre-productif d'une jurisprudence négative, qui entraîneraient 
à la fois une perte de notoriété et un échec en termes de justice sociale. Cet ordre de grandeur (de l'efficacité et du professionnalisme) s'accorde mal avec le monde civique (de l'égalité et de la légalité) et interroge les juristes du CECLR et de l'IEFH sur le sens de leur travail. Ils doivent bien admettre que l'inégalité n'est pas la même pour tout le monde, compte tenu du degré d'objectivation des situations vécues et rapportées par les plaignants.

Moi j'ai pris l'option de toujours croire ce que les gens me disent. Leur récit, c'est la manière dont ils ont vécu la situation, c'est ce qui compte finalement. Mais quand quelqu'un n'a ni témoins, ni preuves écrites de la discrimination, on est impuissant, alors que si les personnes peuvent apporter des preuves, même si les faits sont moins graves pour la personne, alors on peut agir. C'est difficile d'expliquer aux gens qu'une action en justice requiert des éléments de preuve, alors qu'ils sont dans une situation de détresse (Conseiller juridique, CECLR).

\section{Une intervention connexionniste}

Enfin, une série d'autres critères entrent en considération : favoriser, au sein de la population et des acteurs qui interviennent en droit du travail, une plus grande prise de conscience de certaines questions; renforcer la capacité d'action de certains groupes marginalisés; élaborer des partenariats avec des acteurs de la société civile tels que syndicats, associations, etc.

L'un de nos objectifs premiers, c'est vraiment de faire comprendre aux magistrats, aux avocats, aux syndicats, vraiment les premiers interlocuteurs en droit social, ce qu'est une discrimination entre les femmes et les hommes, en ce compris les transsexuels et les femmes enceintes. Ça c'est vraiment un peu notre objectif (Conseillère juridique, IEFH).

Ces critères relèvent plus spécifiquement de la cité par projets (Boltanski, Chiapello, 1999, 161-192), comme le travail de socialisation ou de conscientisation de groupes professionnels (magistrats et avocats), lélaboration de partenariats avec les syndicats et le monde associatif, le renforcement des capacités d'action (empowerment) de certains groupes marginalisés, etc. L'enjeu est ici la constitution et l'extension de réseaux d'acteurs, la création et la prolifération de liens, la mobilisation de partenaires dans le cadre de multiples projets de promotion de l'égalité. Notons que cet univers de justification est dissonant avec celui du renom: en effet, le connexionnisme implique un certain effacement des frontières organisationnelles et professionnelles ainsi qu'une forme de renoncement à la notoriété et à l'autolégitimation, au profit d'une mobilisation collective, de victoires partagées. Notons également que cet enjeu d'empowerment de groupes marginaux peut s'avérer contradictoire avec la «sélection sociale» des cas qu'induit inévitablement le principe de robustesse du dossier.

En sens inverse, les mêmes arguments sont mobilisés par les conseillers juridiques pour justifier une décision de refus d'aller en justice: l'incertitude sur la qualification de discrimination, le manque d'éléments de preuve et de témoignages, le risque de créer une jurisprudence négative ou encore le fait qu'il ne s'agit pas d'une question qui permettra de «faire jurisprudence». 


\section{De la sélection à la recherche de cas}

Dans certains cas, le processus de choix s'inverse. Les organismes de promotion de l'égalité ne procèdent alors plus à une sélection mais se lancent dans la recherche active de "cas exemplaires» susceptibles de créer une jurisprudence positive autour de questions précises de droit. Ils ont alors une démarche proactive de sollicitation des travailleurs plus que de sélection.

Ça faisait longtemps qu'on cherchait un beau cas de discrimination homme-femme en raison de l'ostéoporose. On en a peut-être enfin un qui va pouvoir faire jurisprudence! (Conseillère juridique, IEFH).

Lorsque la victime est soutenue dans une action en justice par le CECLR ou l'IEFH, des contradictions sont susceptibles d'apparaître entre la demande de compensation individuelle exprimée par la victime et la revendication de principe de ces organismes. En effet, il est important pour ces derniers que la procédure en justice arrive à son terme et qu'il n'y ait pas de transactions avant le jugement. Ils incitent donc la victime à poursuivre la procédure, même lorsque celle-ci souhaite l'abandonner avant le jugement.

On essaie de faire en sorte que la victime ne nous lâche pas en cours de route. Par exemple, on a eu un cas, lors de l'entretien, on lui a bien dit qu'on devait vraiment utiliser son cas pour aller plus loin et faire modifier la législation. Donc ce n'est pas parce que la victime obtient son indemnisation qu'on doit interrompre l'action en justice. On ne veut pas que ça soit une transaction, parce que sinon ça risque de ne pas faire évoluer la jurisprudence (Conseillère juridique, IEFH).

Cet exemple illustre bien les difficultés inhérentes au recours aux juridictions dans le domaine de la lutte contre les discriminations. La demande de justice individuelle exprimée par les travailleurs qui s'estiment victimes de discrimination se trouve ainsi confrontée à l'intérêt collectif défendu par les organismes de promotion de l'égalité des chances qui cherchent à produire de la jurisprudence novatrice dans le domaine des discriminations. En clair, la «petite affaire privée» rapportée au guichet de ces organismes par un profane est assez rapidement réinterprétée, traduite, par quatre ordres de discours partiellement discordants qui la rendent publique: celui de l'intérêt collectif de la société civile (le respect du principe de non-discrimination et la volonté de faire jurisprudence), celui de l'intérêt organisationnel du CECLR et de l'IEFH (la reconnaissance des organismes de promotion de l'égalité), celui de l'intérêt professionnel (le résultat performant de leur intervention), celui de l'intérêt associatif (la création et l'activation de connexions). Lorsque les conseillers juridiques parviennent à agencer ces quatre ordres de discours, leurs organismes mènent des actions de strategic litigation.

Il s'agit dès lors, pour ces organismes, de ne pas passer à côté de cas qui pourraient créer une jurisprudence significative. Dans certaines affaires, l'argument de la discrimination n'a été mis en avant ni par les parties, ni par leurs avocats. Alors que ces cas auraient pu constituer des «cas exemplaires» parce qu'ils auraient pu s'inscrire dans un domaine du droit des discriminations où la jurisprudence est faible ou inexistante, la décision des parties de se référer à d'autres législations que celles de la non-discrimination empêche de 
créer une jurisprudence positive. Une avocate du travail spécialisée en droit des discriminations témoigne sa déception quand elle apprend qu'une affaire qui aurait pu faire jurisprudence n'a pas été traitée sous l'angle de la discrimination.

On retrouve pas mal de dossiers où on se dit, a posteriori, quand c'est publié: «Bon sang il y avait matière!» [...] Il y a un arrêt de la Cour du travail d'Anvers en matière d'un non-renouvellement de contrat. La travailleuse était enceinte et elle fait une fausse couche. Elle se fait licencier à la suite de sa fausse couche et elle considère que le licenciement est lié à sa fausse couche. [...] Son avocat a le réflexe "loi de 71 , protection de la maternité». Intelligemment, il va essayer d'interpréter la notion de maternité de manière extensive afin d'inclure la fausse couche et d'étendre la protection de la femme enceinte qui n'est plus enceinte. Mais il s'arrête là dans son raisonnement. Il ne va pas plus loin et ne se dit pas «il y a une discrimination sur base du sexe, parce que toute question liée à la maternité est discriminante sur base du sexe ». Du coup, la Cour du travail adopte une interprétation restrictive de la notion de maternité et refuse d'assimiler une fausse couche à une période de maternité pour la protection, donc la dame a perdu son procès (Avocate en droit du travail).

«Bon sang, il y avait matière!», s'exclame cette avocate. Certains dossiers susceptibles de créer une jurisprudence positive dans le champ du droit contre les discriminations échappent aux organismes de promotion de l'égalité et aux avocats qui se sentent concernés par la question des discriminations. Cette frustration face à ces « cas manqués » invite alors, au-delà de la sélection des cas qui sont soumis à l'expertise de ces organismes, à adopter une attitude proactive de recherche de dossiers potentiellement exemplaires. Au lieu de se prononcer ou de ne pas se prononcer sur une question qui leur est soumise, il s'agit de solliciter une demande de la part des travailleurs. Dans le cas suivant, la conseillère juridique prend connaissance, dans la presse grand public, d'un cas qui mériterait, de son point de vue, d'être traité sous l'angle de la discrimination en raison du genre. Elle se demande alors quelle position adopter lorsque la personne qui s'estime victime de discrimination n'a pas sollicité son institution pour faire valoir ses droits:

Un jour, on a lu un article de presse à propos d'une travailleuse qui était statutaire en Communauté flamande [...]. Cette dame prend un congé pour convenance personnelle et travaille comme contractuelle dans des projets d'immersion en Communauté française. À ce moment-là, elle tombe enceinte et elle n'est pas indemnisée pendant son congé de maternité. Pourquoi ? Parce qu'elle a cotisé comme statutaire mais pas comme contractuelle. Voilà donc une carence législative claire, qui est contraire, selon nous [...] au droit européen, etc. Et donc nous, on voit cet article de presse. On se dit «Qu'est-ce qu'on fait?». [...] La personne n'est jamais venue chez nous, mais on se rend compte qu'elle a sollicité le médiateur de la Communauté française, alors que c'est un problème de genre, que le médiateur de la Communautéfrançaise ne peut rien faire. Que faire? On ne peut quand même pas aller chercher les plaignants par la peau du dos s'ils ne viennent pas à nous. Dans ce cas, finalement, j'ai demandé au Médiateur de dire à cette dame de prendre contact avec nous, ce qu'elle a fait (Conseillère juridique, IEFH). 
La strategic litigation apparaît comme une forme de jugement équilibré (Eymard-Duvernay, Marchal, 1997). Ce processus de sélection et de recherche de cas exemplaires constitue, aux yeux des conseillers, une voie possible de compromis au carrefour de ces différents ordres de discours ou registres de justification. Ce concept relativement flou et hautement mobilisateur permet au collectif des deux organisations étudiées d'esquisser une stratégie commune (de sélection et de recherche de cas exemplaires) qui intègre de multiples appuis conventionnels de l'action pour la défense des travailleurs victimes de discrimination. L'équilibre du jugement est une garantie de qualité pour une intervention juste au regard du droit, ce qui permet aux conseillers juridiques de devenir les garants d'un principe d'égalité; reconnue dans de nombreux auditoires (le grand public, la profession juridique, le monde du travail, le mouvement associatif, syndical, etc.), ce qui permet d'accroître la légitimité et la visibilité de leurs organisations; performante, en raison du professionnalisme et de l'expertise juridique, ce qui permet de ne pas prendre des risques inutiles dans des dossiers trop peu solides et de maximiser les chances d'une jurisprudence positive; enfin, connexionniste, grâce aux multiples contacts que ces organismes développent avec leur environnement, ce qui permet de promouvoir et de pérenniser la mobilisation d'alliés pour des actions collectives.

\begin{tabular}{|l|l|l|l|l|}
\hline Critères de choix & $\begin{array}{l}\text { Critères légaux } \\
\text { de discrimination } \\
\text { et faiblesse de la } \\
\text { jurisprudence }\end{array}$ & $\begin{array}{l}\text { Domaines } \\
\text { prioritaires }\end{array}$ & $\begin{array}{l}\text { Solidité et } \\
\text { robustesse }\end{array}$ & $\begin{array}{l}\text { Prise en compte } \\
\text { de l'impact du } \\
\text { choix sur la société }\end{array}$ \\
\hline Arguments & $\begin{array}{l}\text { Intervention juste } \\
\text { au regard du droit }\end{array}$ & $\begin{array}{l}\text { Intervention } \\
\text { reconnue }\end{array}$ & $\begin{array}{l}\text { Intervention } \\
\text { performante }\end{array}$ & $\begin{array}{l}\text { Intervention } \\
\text { connexionniste }\end{array}$ \\
\hline $\begin{array}{l}\text { Registres de } \\
\text { justification }\end{array}$ & $\begin{array}{l}\text { Civique: } \\
\text { égalité et légalité }\end{array}$ & $\begin{array}{l}\text { Opinion: } \\
\text { visibilité et } \\
\text { légitimité }\end{array}$ & $\begin{array}{l}\text { Industriel: } \\
\text { efficacité et } \\
\text { professionnalisme }\end{array}$ & $\begin{array}{l}\text { Cité par projets: } \\
\text { partenariats et } \\
\text { réseaux }\end{array}$ \\
\hline
\end{tabular}

\section{Conclusion}

À partir d'une étude des processus de filtrage des dossiers, cet article met en évidence les modes d'action des organismes de promotion de l'égalité et de lutte contre les discriminations et, particulièrement, le recours à l'arène judiciaire. Face aux multiples victimes, réelles ou supposées, qui se présentent à eux, ces organismes mettent en place un processus de sélection qui repose sur une multitude d'arguments déployés par les conseillers juridiques: les deux premiers groupes de critères touchent au droit en tant que tel et aux opportunités de renforcer son application, voire de le transformer (monde civique); d'autres renvoient aux stratégies de ces organismes et à leur souhait d'accroître leur légitimité et leur visibilité dans le domaine de la lutte contre les discriminations (monde de l'opinion); d'autres arguments sont orientés vers la constitution de partenariats, l'extension de réseaux d'acteurs, la mobilisation collective autour des questions traitées par ces organismes (monde connexionniste) ; d'autres, enfin, sont des arguments de performance, fondés sur l'expertise professionnelle et l'anticipation des chances d'obtenir une jurisprudence positive (monde 
industriel). La strategic litigation apparait alors comme une forme de compromis entre ces «grandeurs» hétérogènes que sont l'intervention juste, le renom de ces organismes, la performance de leur activité et la mobilisation d'alliés.

Ce double processus de sélection et de recherche active de cas exemplaires s'inscrit dans une stratégie plus générale de transformation des droits des travailleurs par le biais d'actions en justice. La démarche de ces organismes de promotion de l'égalité, des avocats et des associations qui interviennent dans le domaine des discriminations consiste à utiliser les juridictions pour transformer des politiques ou législations existantes. Alors que le système belge a traditionnellement rejeté le principe de l'accès aux cours et tribunaux pour revendiquer des droits sociaux et politiques, les actions menées par les organismes de promotion de l'égalité témoigneraient d'une transformation du recours aux juridictions et de modes d'action des organisations de promotion des droits. En effet, en Belgique, les associations, organisations ou individus qui revendiquaient des modifications des politiques ou législations en vigueur se sont plutôt orientés vers une stratégie de mobilisation du pouvoir législatif que judiciaire, les actions de lobbying auprès de parlementaires ayant plus de chance d'aboutir à un résultat en leur faveur. Cependant, dans le domaine de la lutte contre les discriminations, on peut observer que la strategic litigation est devenue une pratique, sinon courante, du moins significative.

En deux mots, notre objectif ici, c'est ce qu'on appelle la strategic litigation. C'est le mot à la mode ici. C'est vraiment par rapport à ce critère qu'on sélectionne les dossiers qui vont en justice. La sélection, ça fait parfois l'objet de débats en réunion... parce qu'on essaie d'adopter une ligne de conduite pour choisir les dossiers. [...] Mais après, il faut voir au cas par cas (Conseiller juridique, CECLR).

Ce modèle de la strategic litigation provient des pays de common law dans lesquels les cours et tribunaux ont joué traditionnellement un rôle plus important dans la promotion de la non-discrimination et, de manière plus générale, dans la promotion des droits. L'importation apparente de ces modes d'action issus de pays anglo-saxons pose la question de la transnationalisation et la globalisation du droit et des modes de recours aux juridictions (Merry, 1992; Delpeuch, Vassileva, 2009). L'appartenance à l'Union européenne tendraitelle à élargir les modalités de recours aux cours et tribunaux et les modes de promotion des droits en Belgique? L'Union européenne incite les États-membres à mettre sur pied des organismes destinés à traiter spécifiquement la question des discriminations. Le réseau européen Equinet, qui réunit les membres des agences gouvernementales de promotion de l'égalité afin de fédérer les actions entreprises au sein de chaque État et de développer des stratégies communes, incite également ces organismes à réfléchir aux stratégies à adopter dans le cadre de la défense d'un dossier en justice, tant devant les juridictions nationales qu'internationales, la Cour européenne de Justice ou la Cour européenne des Droits de l'Homme.

La strategic litigation en Belgique constituerait alors un cas singulier de mimétisme institutionnel (Hassenteufel, 2005) ou d'isomorphisme mimétique (Powell, DiMaggio, 1991) ${ }^{13}$.

13 Rappelons que cette théorie néo-institutionnaliste soutient l'idée selon laquelle les organisations ont tendance à s'imiter les unes les autres en contexte d'incertitude; la diffusion horizontale de modèles, d'instruments, de pratiques, résulterait, selon ces auteurs, de la circulation des individus entre les institutions (d'un même pays ou de contextes nationaux différents). 
En effet, rien ne prouve aujourd'hui que cette stratégie soit le produit d'une harmonisation transnationale de l'usage des cours et tribunaux. Il nous faut donc renoncer à une conception mécaniste des processus de transfert. Par contre, notre étude de cas montre que les juristes du CECLR et de l'IEFH s'inspirent de ce modèle anglo-saxon, l'adaptent plus que ne l'adoptent: ce modèle leur offre des ressources cognitives et normatives pour faire tenir ensemble des enjeux contradictoires au sein de leur organisme, pour concilier des grandeurs irréductibles face au public, pour assurer des missions officielles qui ne se limitent pas à l'application du droit mais qui impliquent un travail culturel d'interprétation de nouvelles orientations de sens, proposées par les institutions européennes, tant dans la sphère juridique que dans le monde vécu des personnes. Ce travail de mise en cohérence des significations multiples du droit (en particulier, des concepts de non-discrimination et d'égalité de chances) passe nécessairement par des délibérations, des communications, des actions collectives, mettant en jeu syndicats, associations, professionnels du droit, entreprises, etc. Dans le cadre de réseaux européens, comme Equinet, ces juristes se familiarisent au modèle anglo-saxon de la strategic litigation, dans une logique d'émulation et d'inspiration, et l'adoption non contraignante ou la traduction qu'ils font de cette stratégie, en Belgique, contribue à l'ouverture de nouveaux «mondes possibles» (De Munck, Orianne, 2008).

Il faut cependant mentionner que, si cette pratique de la strategic litigation est courante au sein des organismes de promotion de l'égalité, son impact sur la vie des juridictions ne doit pas être surestimé. En effet, seuls quelques avocats spécialisés en droit du travail et quelques juristes travaillant pour des syndicats recourent à cette pratique. Néanmoins, les cas observés montrent une évolution sensible des modes de recours aux tribunaux. On peut donc affirmer que le succès de cette pratique en Belgique résulte du fait qu'elle est soutenue et encouragée par les organismes de promotion de l'égalité. Ces derniers éprouvent le besoin de recourir à cette stratégie pour affirmer leur légitimité et négocier leur place en tant qu'acteurs émergents, raison pour laquelle cette pratique se pose en alternative aux usages traditionnels de recours aux cours et tribunaux, désormais utilisés pour promouvoir des transformations des politiques et législations en vigueur. Ceci viendrait confirmer la thèse selon laquelle «la réception des greffes exogènes dans un système juridique local est moins tributaire des efforts déployés par les prescripteurs que des éventuelles stratégies d'instrumentalisation mises en ouvre par les destinataires » (Delpeuch, Vassileva, 2009,399; Dezalay, Garth, 2002). La proximité physique de l'Union européenne à Bruxelles constitue également un facteur important qui incite ces organismes belges à montrer l'exemple en matière de traitement des discriminations.

La pratique de sélection étudiée ici concerne le champ du droit social, néanmoins, l'enjeu de cette recherche est, plus généralement, de réfléchir à l'exemplarité de la situation vécue par une victime de discrimination et de proposer de nouvelles pistes de recherche, encore peu explorées, sur les modes de recours aux cours et tribunaux dans les pays de tradition de droit civil, en comparaison avec les pays de droit anglo-saxon. Si de récents travaux posent la question des usages stratégiques de l'arène judiciaire, une analyse approfondie des enjeux et conséquences de ces mobilisations de la justice reste largement à construire.

Aude Lejeune CNRS/CERAPS aude.lejeune@univ-lille2.fr ISHS, Université de Liège jforianne@ulg.ac.be 


\section{Bibliographie}

AGRIKOLIANSKY E., 2003, Usages choisis du droit. Le service juridique de la Ligue des droits de l'Homme (1970-1990) entre politique et raison humanitaire, Sociétés Contemporaines, 52, 61-84.

ALTER K., VARGAS J., 2000, Explaining Variation in the Use of European Litigation Strategies: European Community Law and British Gender Equality Policy, Comparative Political Studies, 33, 452-482.

BERENI L., CHAPPE V.-A., 2011, La discrimination, de la qualification juridique à l'outil sociologique, Politix, 94, 9-34.

BOLTANSKI L., CHIAPELLO È., 1999, Le nouvel esprit du capitalisme, Paris, Gallimard.

BOLTANSKI L., THÉVENOT L., 1991, De la justification. Les économies de la grandeur, Paris, Gallimard.

BOUWEN P., MCCOWN M., 2007, Lobbying versus Litigation: Political and Legal Strategies of Interest Representation in the European Union, Journal of European Public Policy, 14, 3, 422-443.

BRIBOSIA E., RORIVE I., 2003, Les nouvelles lois antidiscriminatoires: quelles avancées pour la Belgique en matière de lutte contre le racisme?, Année sociale, 83-94.

CARADEC V., LEFRANCOIS C., POLI A., 2009, Quand la discrimination et la diversité se déclinent selon l'âge: émergence, appropriations, ambivalences, Cahiers Internationaux de Sociologie, 2, 127, 223-245.

CASE R. E., GIVENS T., 2010, Re-engineering Legal Opportunity Structures in the European Union? The Starting Line Group and the Politics of the Racial Equality Directive, Journal of Common Market Studies, 48, 2, 221-241.

CHAPPE V.-A., 2010, La qualification juridique est-elle soluble dans le militantisme? Tensions et paradoxes au sein de la permanence juridique d'une association antiraciste, Droit et Société, 76, 543-567.

COMMAILLE J., 2000, Territoires de justice: une sociologie politique de la carte judiciaire, Paris, PUF, coll. «Droit et Justice».

COMMAILLE J., DUMOULIN L., 2009, Heurs et malheurs de la légalité dans les sociétés contemporaines. La sociologie politique de la «judiciarisation », L’Année Sociologique, 59, 1, 63-107.

CONANT L., 2006, Individuals, Courts, and the Development of European Social Rights, Comparative Political Studies, 39, 76-100.

DE MUNCK J., ORIANNE J.-F., 2008, Droits sociaux et mondes possibles. L'exemple du droit européen au congé parental, Raisons Pratiques, 18, 263-280.

DELPEUCH T., VASSILEVA M., 2009, Contribution à une sociologie des entrepreneurs internationaux de transferts de réformes judiciaires, L'Année Sociologique, 59, 2, 371-402.

DEZALAY Y., GARTH B., 2002, La mondialisation des guerres de palais: la restructuration du pouvoir d'État en Amérique latine entre notables du droit et 'Chicago Boys', Paris, Le Seuil.

EYMARD-DUVERNAY F., MARCHAL E., 1997, Façons de recruter, Paris, Métailié.

FASSIN D., 2002, L'invention française de la discrimination, Revue française de Science Politique, 52, 4, 403-423.

GALANTER M., 1974, Why the 'Haves' Come out Ahead: Speculations on the Limits of Legal Change, Law and Society Review, 9, 95-160.

GARCIA VILLEGAS M., 2009, Champs juridiques et sciences sociales en France et aux États-Unis, L'Année Sociologique, 59, 1, 29-62.

GUILLAUME C., POCHIC S., 2012, Le recours aux tribunaux, une stratégie payante pour l'égalité salariale en Grande-Bretagne?, Séminaire du RT18: Relations professionnelles et discriminations au travail: quels usages du droit?, Paris.

GUIRAUDON V., 2009, Equality in the Making: Implementing European non-Discrimination Law, Citizenship Studies, 13, 5, 527-548.

HANDLER J., HOOLINGSWORTH E., ERLANGER H., 1978, Lawyers and the Pursuit of Rights, New York, Academic Press.

HASSENTEUFEL P., 2005, De la comparaison internationale à la comparaison transnationale. Les déplacements de la construction d'objets comparatifs en matière de politique publique, Revue Française de Science Politique, 55, 1, 113-132. 
INTERIGHTS, EUROPEAN ROMA RIGHTS CENTRE, 2004, Strategic Litigation of Race Discrimination in Europe: from Principles to Practice [http://www.migpolgroup.com/public/docs/57.StrategicLitigationofRace DiscriminationinEurope-fromPrinciplestoPractice_2004.pdf].

ISRAËL L., 2001, Usages militants du droit dans l'arène judiciaire: la cause lawyering, Droit et Société, 49, 793-824.

ISRAËL L., 2009, L'arme du droit, Paris, Presses de Sciences Po.

KELEMEN D., 2011, Eurolegalism: The Transformation of Law and Regulation in the European Union, Cambridge, Harvard University Press.

LEJEUNE A., 2011, Les professionnels du droit comme acteurs politiques. Bilan de la littérature nord-américaine et enjeux de l'importation de ces travaux en Europe continentale, Sociologie du Travail, 53, 2, 216-233.

LEJEUNE A., 2014, Mobilisations du droit, dans ou hors des tribunaux? La lutte contre les discriminations à l'emploi en Belgique et en Suède, in BAUDOT P.-Y., REVILLARD A., L'État des droits. Politiques des droits et pratiques des institutions, Paris, Presse de Sciences, Colloque L'État des droits. Pratiques des droits dans l'action publique, Paris.

LOCHAK D., 1989, Les usages sociaux du droit, Paris, PUF.

MATHER L., 2009, Bringing Lawyers Back, in MILLER M. (ed.), Exploring Judicial Politics, New York \& Oxford, Oxford University Press, 48-63.

MATHIEU L., 2001, Mobilisations de prostituées, Paris, Belin.

MCCANN M., 1994, Rights at Work: Pay Equity Reform and the Politics of Legal Mobilizations, Chicago \& Londres, The University of Chicago Press.

MEMMI D., 1989, Demande de droit et vide juridique: les juristes aux prises de la construction de leur propre légitimité, in CURAPP, Les usages sociaux du droit, Paris, PUF, 13-31.

MERRY S. E., 1992, Anthropology, Law, and Transnational Processes, Annual Review of Anthropology, 21, 357-379.

MILlER M. (ed.), 2009, Exploring Judicial Politics, New York \& Oxford, Oxford University Press.

MILLER R. E., SARAT A., 1981, Grievances, Claims and Disputes: Assessing the Adversary Culture, Law E Society Review, 15, 3, 525-566.

PELISSE J., 2005, A-t-on conscience du droit? Autour des Legal Consciousness Studies, Genèses, 59, 114-130.

PELISSE J., 2009, Judiciarisation ou juridicisation? Usages et réappropriations du droit dans les conflits de travail, Politix, 86, 73-96.

PERROUTY P.-A., RORIVE I., 2005, Réflexions sur les difficultés de preuve en matière de discriminations, Revue du droit des étrangers, 133, 161-175.

POWELL W.W., DIMAGGIO P.J. (Eds), 1991, The New Institutionalism in Organizational Analysis, Chicago, University of Chicago Press.

REVILLARD A., 2007, Entre arène judiciaire et arène législative: les stratégies juridiques des mouvements féministes au Canada, in COMMAILLE J., KALUSZYNSKI M. (dir.), La fonction politique de la justice, Paris, La Découverte, Coll. «Recherches», 145-164.

RINGELHEIM J., 2010, Les transformations du droit de la non-discrimination, in HERMAN G., LÉONARD E., REMAN P. (dir.), Travail, inégalités et responsabilité, Louvain-la-Neuve, Presses universitaires de Louvainla-Neuve.

ROSENBERG G., 1993, The Hollow Hope: Can Courts Bring About Social Change?, Chicago, The University of Chicago Press.

SARAT A., SCHEINGOLD S., 1998, Cause Lawyering: Political Commitments and Professional Responsibilities, New York, Oxford University Press.

SCHEINGOLD S., 2004 [1974], The Politics of Rights: Lawyers, Public Policy and Political Change, New Haven \& Londres, Yale University Press.

STURM S., 2001, Second Generation of Employment Discrimination: a Structural Approach, Columbia Law Review [on line], 101, 1 [http://ssrn.com/abstract=244407].

TERRAZ I., JAOUL-GRAMMARE M., 2012, Diversité et évolution de la syndicalisation en Europe, Bulletin de l'Observatoire des politiques économiques en Europe, 26, 35-39. 
VANHALA L., 2009, Anti-discrimination policy actors and their use of litigation strategies: the influence of identity politics, Journal of European Public Policy, 16, 5, 738-754.

VAUCHEZ V., 2001, Entre droit et sciences sociales. Retour sur l'histoire du mouvement Law and Society, Genèses, 45, 134-149.

WILLEMEZ L., 2003, Engagement professionnel et fidélité militante. Les avocats travaillistes dans la défense judiciaire des salariés, Politix, 62, 145-164.

\section{Summary}

Strategic litigation is one of the methods used by equal opportunity agencies in Europe to promote rights. In relation to organisations, this article aims to show how and under which circumstances strategic litigation is deemed relevant to fight employment discrimination in Belgium and on which grounds and arguments exemplary cases are chosen. The article also highlights possible changes in the use of courts and the judiciary.

\section{Zusammenfassung}

Der strategische Rückgriff auf die Justice ist eine Methode, die von Organisationen im Bereich der Chancengleichheit in Europa eingesetzt wird. Aus einer Organisationperspektive untersucht der Artikel die Bedingungen und Modalitäten, mit denen diese Methode in Belgien im Kampf um Chancengleichheit praktiziert wird, die Kriterien und Argumente, mit denen exemplarische Fälle ausgewählt werden und dessen mögliche Transformationen, die diese Fälle durch Gerichte und die juristische Arena erfahren.

\section{Sumario}

El uso estratégico a los tribunales, llamado strategic litigation, forma parte de las estrategias de los organismos europeos de promoción de la igualdad de oportunidades. Centrándose en el nivel organizativo, este artículo examina los medios y condiciones de esta práctica en el contexto de la lucha contra la discriminación en Bélgica, los criterios y argumentos que se utilizan para seleccionar los casos considerados como ejemplares, así como las posibles transformaciones que se producen en el uso de los tribunales. 


\section{Annexe}

\section{Le Centre pour l'égalité des chances et la lutte contre le racisme (CECLR)}

\section{Missions}

- Être un organisme consultatif au service des citoyens dans des situations de discrimination.

- Aider les citoyens à travers une série d'outils comme par exemple la conciliation ou la médiation.

- Analyser différents phénomènes [...] à travers diverses actions avec différents acteurs.

- Assurer des missions de prévention, d'information, de sensibilisation et de formation auprès des décideurs, des associations nationales ou internationales, des citoyens, etc.

- Susciter, conseiller et recommander les autorités publiques [...]

- Établir de nombreux partenariats [...] afin de créer un véritable débat fructueux et ancré dans la réalité, et de créer des actions ciblées.

\section{Nombre de cas traités}

En 2009, 2888 signalements ont donné lieu au traitement de 1877 dossiers, dont 381 dossiers concernent I'emploi et le travail (176 concernent l'accès à l'emploi, 123 les conditions de traitement au travail, 53 les licenciements, 9 «autres») 1 .

\section{Composition}

Une centaine de personnes travaillent au CECLR au sein des services discriminations, égalité des chances, migration, pauvreté et gestion interne.

\section{L'Institut pour l'égalité des femmes et des hommes (IEFH)}

\section{Missions $^{2}$}

- Effectuer, développer, soutenir et coordonner les études et recherches en matière de genre et d'égalité $[\ldots]$

- Adresser des recommandations aux pouvoirs publics [...] ainsi qu'aux institutions privées

- Organiser le soutien aux associations [...]

- Aider toute personne sollicitant une consultation sur le contenu et l'étendue de ses droits et obligations $[\ldots]$

- Agir en justice dans les litiges auxquels pourrait donner lieu l'application des lois pénales et autres lois dont l'objet spécifique est de garantir l'égalité des femmes et des hommes

- Produire et fournir toute information, documentation, et archives utiles dans le cadre de son objet

- Recueillir et publier les données statistiques et les décisions juridictionnelles [...]

- Demander à l'autorité compétente de s'informer et de tenir informé celle-ci des résultats de l'analyse des faits dont il est question. [...]

- Élaborer une structure de réseau avec les différents acteurs dans le domaine de l'égalité des femmes et des hommes

\section{Nombre de cas traités par an}

En 2009, 196 notifications et 491 appels téléphoniques ont donné lieu au traitement de 150 dossiers dont 97 dossiers concernent l'emploi et le travail ${ }^{3}$.

\section{Composition}

35 personnes travaillent à I'IEFH au sein des cellules et services gender mainstreaming, recherche, violence, juridique, gestion interne et communication.

\footnotetext{
1 Rapport annuel 2009 du CECLR.

2 Art. 4, loi du 16 décembre 2002.

3 Rapport annuel 2009 de I'IEFH.
} 